\title{
INTEGRACIÓN ECONÓMICA REGIONAL LIBERAL E INSERCIÓN EN LA ECONOMÍA MUNDIAL: UNA VISIÓN CRITICA A PARTIR DE UN ESTUDIO DEL COMERCIO DE AMERICA DEL SUR 1985-2009 ${ }^{(*)}$
}

\author{
LIBERAL REGIONAL ECONOMIC INTEGRATION AND INSERTION IN THE \\ WORLD A ECONOMY: \\ A CRITICAL VIEW FROM A STUDY OF TRADE IN SOUTH AMERICA 1985- \\ 2009
}

\begin{abstract}
Alexis Saludjian ${ }^{(* *)}$
Universidad Federal do Rio de Janeiro, Rio de Janeiro (RJ), Brasil.
\end{abstract}

Resumen: En el inicio de los años 1990, los países de America del Sur estaban buscando modelos de desarrollo alternativos a los que fueron implementados en los años 1960-1980. El Regionalismo Abierto fue una manera de incluir las nuevas teorías económicas sobre comercio internacional y el desarrollo a la temática de la integración regional. Presentaremos un estudio empírico sobre la inserción de América del Sur mobilizando datos do período 1985-2009 con una distinción entre productos primarios e industriales y también según el tipo de tecnología utilizada además de si están o no relacionados con recursos naturales o intensivos en trabajo.

Palabras Claves: Regionalismo abierto; integración regional; inserción internacional.

Abstract: At the beginning of the 1990s, Southern American countries were seeking alternative development models which were implemented in the period 1960-1980. Open Regionalism was an alternative to include the new economic theories of international trade and development to the issue of regional integration. This paper presents an empirical study on the insertion of South America in the world economy. We will use data for the period 1985-2009 with a distinction between primary and industrial products, between type of technology used and between natural resources and labor intensive godos.

Keywords: Open regionalism; Regional integration; International insertion.

${ }^{*}$ ) Parte de este trabajo fue presentado en el seminario "Red globalización financiera y desarrollo sustentable; $2^{\circ}$ coloquio internacional; opciones frente a la crisis global: estrategias para un desarrollo sustentable". 26-27 de marzo 2012. UNAM, UAM, UABCS, Mexico DF.

$(* *)$ Doctor, Profesor del Instituto de Economía de la Universidad Federal do Rio de Janeiro (IE/UFRJ). Investigador asociado del CEPN-Paris 13 (Francia). E-mail: <saludjian@ie.ufrj.br.> Recebido en: 02.10.2013, aceptado en: 15.11.2013 


\section{INTRODUCCIÓN}

En el inicio de los años 1990, al salir de la "década perdida", los países de America del Sur estaban buscando modelos de desarrollo alternativos a los que fueron, correctamente o no, implementados en los años 1960-1980. El peso de la deuda y sus consecuencias económicas y sociales fueron tan importantes que la opción de un apoyo público fuerte parecía descartada e irrelevante para la ortodoxia prevaleciente en esta época.

Una vía alternativa y transformadora más radical estaba descartada a causa del fin de la Guerra Fría y frente al capitalismo triunfante. El Regionalismo Abierto o "Nuevo Regionalismo" fue una manera de incluir las nuevas teorías económicas sobre comercio internacional y el desarrollo a la temática de la integración regional que estaba aconteciendo en todas las regiones del mundo: la reconfiguración de los espacios económicos y geopolíticos fue muy fuerte y no únicamente en las Américas. Presentaremos en este trabajo un estudio empírico sobre la inserción de América del Sur.

Para esto, movilizamos datos de la UN-COMTRADE durante el período 1985-2009 con una distinción entre productos primarios e industriales y también según el tipo de tecnología utilizada (baja, media o alta) además de si están o no relacionados con recursos naturales o intensivos en trabajo.

Así, será posible verificar el tipo de participación de América del Sur en el comercio mundial y si la región se benefició de esta política económica (Regionalismo Abierto) para mejorar efectivamente y substancialmente su inserción en la economía mundial. Podremos también tener algunos elementos para pensar qué tipo de modelos de desarrollo genera este tipo de inserción y si se redujo la heterogeneidad estructural de la región.

\section{RESULTADOS EMPÍRICOS DE LA INTEGRACIÓN ECONÓMICA Y DE LA INSERCIÓN INTERNACIONAL DE AMÉRICA DEL SUR EN LA ECONOMÍA MUNDIAL}

\subsection{COMERCIO, DESARROLLO E INSERCIÓN EN LA ECONOMÍA MUNDIAL}

Este modelo de acumulación basado en las exportaciones (desarrollo para afuera) conoció una breve modificación durante el corto período de la Industrialización por Substitución de las Exportaciones. A partir del final de los años 1980 y principios de los años 1990 vuelve a ser uno de los ejes fundamentales del modelo de acumulación en una economía mundial en profunda mutación con el peso creciente de China y con una reconfiguración de las Cadenas de Valores Globales ${ }^{(1)}$. Estudios recientes muestran que, a pesar del discurso sobre la importancia de las exportaciones y de la inserción más profunda (liberal) en la economía mundial, el peso de las exportaciones en el crecimiento económico fue débil en América Latina cuando lo comparamos con otras regiones, como por ejemplo Asia ${ }^{(2)}$.

(1) No se entra en detalles sobre esta literatura que puede ser encontrada en KAPLINSKY, R.; MoRRIS, M. A handbook for value chain research. Brighton, UK: Institute of Development Studies, 2000.

(2) Ver Mulder (2009) y Palma (2010). 
La Comisión Económica de la Naciones Unidas para el Comercio y el Desarrollo (UNCTAD) trata elementos importantes sobre esta situación en su artículo de septiembre del 2010. En este documento, la UNCTAD, critica la visión liberal (basada en una visión teórica neo-clásica) del desarrollo a través de la abertura/liberalización comercial y de una inserción a cualquier costo en la economía mundial. La UNCTAD critica esta visión en lo que se refiere a la especialización de países en sectores en los cuales la demanda mundial es fuerte o en los cuales el bajo costo de la mano de obra es un elemento esencial de la competitividad de una región. Así, se cuestionan los fundamentos de la visión liberal, que son la base de la (nueva o tradicional) teoría del comercio internacional y del tipo liberal de integración económico regional. Se trata de la relación entre el mercado interno y externo, así como los determinantes de estos diferentes mercados: los países en desarrollo pueden no beneficiarse de una mayor inserción en la economía mundial. De esta manera, sin instituciones referentes a la fuerza de $\operatorname{trabajo}^{(3)}$, sin seguridad social, sin generación propia de bienes de capital o de tecnología, la combinación entre progreso tecnológico, inversión y aumento de la productividad pueden no tener consecuencias sobre la creación de empleo decente, bien remunerado, factores esenciales para una dinamización del mercado interno.

\begin{abstract}
"Between today's developing countries and the countries that industrialized and created labour-market and social-security institutions before the globalization of production and investment, the main differences are not in the macro-economic processes but in the context of corporate decision-making on production and investment. Earlier, such decisions were taken primarily with reference to demand and competition in domestic markets, even when the rest of the world provided markets for some of the increasing production as well as outlets for some labour through migration. By contrast, in most developing countries today such decisions are taken primarily with reference to external demand and global competition. oreover, these countries can import advanced technologies from the North. The problem of combining technological progress, investment and productivity growth with employment creation is more pronounced when labour-saving technology is introduced in an economy that produces neither the capital goods nor the embodied technology. Since this is a typical situation for developing countries, it is even more important for employment creation that productivity gains translate into higher demand for domestically produced goods and services." (UNCTAD, 2010, p. 85)
\end{abstract}

En la visión ortodoxa criticada por la UNCTAD en este artículo, poco importa si existe diferencia entre países más desarrollados, que ya tienen una base de demanda interna robusta y estable (además con una independencia tecnológica) y países menos desarrollados con un elevado nivel de heterogeneidad estructural (y social). La UNCTAD también nota:

"(...) export-led growth strategies tend to lead to relative wage compression, which may seem indispensable for strengthening or maintaining the international competitiveness of producers in any economy. But if many or all countries adopted this strategy it would

(3) Cartera de trabajo, salario mínimo, seguridad del trabajo, mecanismos de seguridad-desempleo, sindicatos y representatividad, etc. 
lead to a "race to the bottom" with regard to wages. This would translate into insufficient growth of workers' purchasing power, which itself is an important determinant for aggregate demand growth and job creation." (UNCTAD, 2010, p. 79)

La relación entre la inserción económica mundial y la especialización de la fuerza de trabajo muestra que la visión ortodoxa de repartición óptima de los factores de producción entre agentes económicos racionales no se reveló sustentable y además no consideró las consecuencias sobre el nivel de salarios ${ }^{(4)}$. Como el mismo trabajo, la UNCTAD apunta que el modelo de desarrollo hacia afuera (export-led) puede promover una carrera de tipo race to the bottom en lo que refiere a los salarios. Esto tiene consecuencias sobre la demanda interna y el poder de compra insuficiente de los trabajadores. Por lo tanto hace que el modelo de desarrollo sea insostenible y muy vulnerable a las oscilaciones de los mercados mundiales ${ }^{(5)}$.

\subsection{ESTRUCTURA Y “CUALIDAD DEL COMERCIO” DE AMERICA DEL SUR (1985-2009):}

En esta sección, presentamos un estudio sobre la evolución del comercio según la composición de las exportaciones/importaciones, basado en datos de la UN-COMTRADE y especialmente en la clasificación CUCI (Clasificación Uniforme para el Comercio Internacional) según la metodología de (S. Lall, 2000) y (UNCTAD, 2002, p. 87-95). A partir de estos datos, el objetivo es entender el papel que juega la integración regional en este proceso y confirmar la hipótesis de "no contribución" de la integración económica liberal e, inclusive, la perversidad de este tipo de regionalismo, que puede empeorar la situación ya precaria de América del Sur en las cadenas globales (y las consecuencias negativas sobre el nivel de formación/calificación/remuneración de la fuerza de trabajo).

Los gráficos (en anexo) indican los saldos comerciales de América del Sur, diferenciando los productos primarios de los productos industrializados y, para estos últimos, en función del contenido tecnológico según la clasificación informada anteriormente ${ }^{(6)}$.

Tenemos que notar que existe una diferencia teórica entre un enfoque ortodoxo y un enfoque crítico en lo que se refiere al estudio sobre el contenido tecnológico de bienes en el comercio internacional. Para los ortodoxos, el contenido en tecnología es importante en la medida en que la producción de alta tecnología aumenta su valor agregado. El hecho de que esta tecnológica sea importada o resulte de un proceso local (o regional) de acumulación de conocimientos, no es muy relevante. Como lo indican las teorías del crecimiento endógeno (P. ROMER, 1990), lo que importa es tener la certeza que el libre comercio pueda garantizar la repartición óptima de los recursos (incluso el "capital humano"). En este tipo de modelos ortodoxos, no importa mucho saber de donde viene la

(4) Ver UNCTAD (2010, p. 78)

(5) No se trata aquí de minimizar la experiencia de ciertos países asiáticos pero si de percibir las diferencias del caso latino-americano. Sobre este punto ver (Salama, 2000). Además, como mostró la crisis de 1997, los países del "milagro asiático" también fueron golpeados por la crisis.

(6) En esta clasificación, el petroleo y los productos derivados no son considerados. Ver detalles en UNCTAD (2002). 
tecnología y si ella es adaptada, costosa, desarticulada del resto del aparato productivo del país o la región y si los deja más vulnerables a las decisiones tomadas en la sedes de las empresas que dieron origen a estas tecnologías (EEUU, Unión Europea, etc. ...).

Al contrario, en este estudio privilegiamos un enfoque en el cual la homogeneidad entre los diferentes niveles de tecnología es importante en cuanto a su origen ${ }^{(7)}$. De esta forma, la planificación de la tecnología permite la aplicación de una política industrial integrada (entre niveles de tecnología y la escala regional), activa y adaptada a las necesidades de los países y de sus poblaciones en función de sus inserciones en la economía mundial. El proceso local de acumulación de conocimientos implica a su vez un esfuerzo superior en educación, investigación y desarrollo y tiene además consecuencias importantes sobre el tipo de desarrollo económico y social.

La lectura de los gráficos y el análisis se desarrollan en tres etapas que serán detalladas como sigue:

Etapa 1: para America del Sur, se analiza la composición de las exportaciones en productos primarios e industrializados ${ }^{(8)}$.

Este primer paso se da tanto para las exportaciones como para las importaciones respectivamente "exportaciones para ..." y "importaciones desde...":

i) Mundo;

ii) América del Sur;

iii) EEUU;

iv) Unión Europea;

v) China ${ }^{(9)}$.

Etapa 2: para las exportaciones y las importaciones, la parte de los bienes industrializados se compone de cuatro categorías:

i) alta intensidad tecnológica;

ii) media intensidad tecnológica;

iii) baja intensidad tecnológica;

iv) intensiva en trabajo y recursos naturales.

Etapa 3: para concluir este estudio, serán analizados los saldos (en volumen y no en porcentajes del total).

(7) "Productivity in an economy and its growth over time are determined by the stock of productive capital, the technology embodied in that capital stock as a result of domestic research and development ( $R E D$ ), access to the foreign technology embodied in imported capital goods (and its expansion over time), as well as the quality of labour resulting from education, vocational training and learning by doing (and its improvement over time)." (UNCTAD, 2010, p. 79.)

(8) Para llegar a 100\% debe considerarse la categoría "otras transacciones", que, para no cargar demasiado a lo gráficos, no representamos en curva en los gráficos.

(9) En la base de datos de la UN-COMTRADE, también están disponibles los destinos/origenes: Japón, Asía-Pacífico, entre otros, pero para no cargar los gráficos, preferimos no representar estas series. 
A continuación será presentado el análisis de los datos de acuerdo a las etapas mencionadas.

A partir de estos resultados es posible tener una visión más detallada del tipo de inserción de América del Sur en la economía mundial, así como del padrón de comercio (y de producción) de la zona. Prestaremos especial atención a las modificaciones y cambios de tendencias, dando énfasis a los periodos en que ocurrieron, para poder determinar si estos cambios están relacionados o no con políticas específicas de integración regional o de abertura comercial.

Preferimos estudiar las relaciones comerciales con los EEUU y la Unión Europea porque son países/zonas que históricamente tuvieron una gran influencia en la configuración del comercio mundial, ya que tuvieron la capacidad de "moldear" las relaciones comerciales a través de su poder de hegemonía (en el caso de los EEUU) y de la antigua relación con América del Sur a partir del período colonial en adelante. Estos países/ zonas centrales en las categorías de la CEPAL original (R. Prebisch) y de la Teoría de la Dependencia ${ }^{(10)}$ a partir del proceso de internacionalización de la producción y del papel de las empresas transnacionales (ETN) tienen la capacidad de articular, a escala mundial, la producción a través de cadenas de valores globales proyectando unidades de producción, fábricas, centros de investigación y desarrollo (I \& D) en cualquier lugar del mundo dependiendo de la estrategia definida en sus sedes.

El caso de China es fundamental para garantizar el crecimiento (o gran parte de este) y mantener la estabilidad macro (liberal) de muchos países de America del Sur a través de la demanda China.

Observaremos primero el comercio con el resto del mundo, luego el comercio con América del Sur (comercio intra-regional). En tercer lugar, veremos el comercio con las potencias del centro (EEUU y Unión Europea) y finalmente con China.

- Comercio de América del Sur con el resto del mundo: en términos generales, la composición de las exportaciones de América del Sur continúa estable desde 1985 con el predominio de productos primarios (en torno de 40\% del total desde los años 2000). Los productos industrializados representan cerca del $30 \%$ de las exportaciones de la región. De este montante, los productos intensivos en tecnología media representan la mayor parte con 30\% a 35\% desde 1996; los productos intensivos en tecnología alta, representan 25\% en 2008 desde que llegaron a más de $30 \%$ en el 2000. Los productos respectivamente intensivos en trabajo y recursos naturales y en baja tecnología representan alrededor del 20\% del total de los bienes industrializados. Las importaciones de America del Sur siguen estando compuestas por más de 70\% de bienes industrializados, mayoritariamente de productos de alta y media tecnología $(40 \%$ para cada categoría). Finalmente, si consideramos el saldo (exportaciones menos importaciones por tipo de producto y por tipo de tecnología), observamos que este es positivo (exportaciones superiores a las importaciones) desde 1985

(10) Ver SANTOS (2000) y MARINI (1977). 
para los productos primarios con un fuerte aumento de este superávit a partir del 2003/2004. En ese mismo período, el déficit (importaciones superiores a las exportaciones) de los productos industrializados resultó mucho más elevado (50 billones de dolares en 2004 y casi 200 billones en 2008/2009). En el mismo período, los saldos negativos de los bienes industrializados con alto y medio nivel tecnológico llegaron a representar 90 y 80 billones de dólares respectivamente.

En esta primera aproximación general del comercio de America del Sur con el resto del mundo, podemos observar que el padrón exportador líquido de productos primarios y de importador líquido de productos industrializados se mantuvo estable durante el período 1985-2009. Desde 2003, el déficit en bienes industriales aumenta aún siendo compensado por el excedente en aumento de los bienes primarios. Como lo veremos más adelante, el peso de China y el período de altos niveles de los precios de las commodities, fueron factores importantes. America del Sur mantuvo su padrón de inserción en la economía mundial desde la década de los 1980/1990 con un pequeña mejora en el contenido de tecnología media y alto ${ }^{(11)}$.

- Comercio de America del Sur con países de la región (o sea comercio intra-regional, indicador de la modificación efectiva del tipo de integración económica sud-americana): Las exportaciones para la región sud-americana, consolidan una predominancia de bienes industrializados de 45\% en 1985 a 55\% en 2008. De este porcentaje, la mayoría son bienes intensivos en tecnología media, que varió de 35\% (en los años 1980) hacia 45\% en los años 1990 y nuevamente desde 2003. Los bienes industrializados de alta tecnología llegaron a representar 30\% en los años 1990 y se estabilizan en torno de 30\% desde 1998. Las otras dos categorías (intensiva en trabajo y recursos naturales y en baja tecnología) representan menos de 15\% cada una. En lo que se refiere a las importaciones de América del Sur en la misma región, se mantiene la tendencia iniciada en los años 1980 con más de 50\% de bienes industrializados y 30\% de bienes primarios. Los bienes industrializados de alta y media tecnología representan $30 \%$ y $40 \%$ respectivamente.

El saldo comercial fue prácticamente nulo en bienes primarios y bienes industriales en los años 1985-1990, se observa un leve superávit en bienes industrializados en los años 1990 que se vuelve deficitario a partir de los años 2000 en los dos segmentos considerados (primarios y industrializados). En 2007, hubo un superávit de 2 billones de dólares en bienes industrializados y de 1,5 billones en bienes primarios, lo cual no se repitió en 2008 y 2009 (cuando nuevamente hubo déficit). Los volúmenes considerados son relativamente reducidos, compatibles con el bajo nivel de comercio intra-regional en América del Sur a pesar de los modelos, acuerdos y discursos sobre la integración económica latino o sud-americana. Una interpretación de este resultado es que el mo-

(11) En este trabajo no discutimos si esta mejoría relativa fue suficiente cuando comparada con la evolución al nivel mundial. Otros trabajos muestran que la mejoría en términos de capacidad de exportaciones de bienes industrializados de media y alta tecnología en America del Sur se mantuvo a un nivel muy inferior a las modificaciones ocurridas en regiones como el Este Asiático. Ver Palma (2010). 
delo de integración abierto (y liberal) de los años 1990 no modificó substancialmente el padrón de comercio a lo largo de las dos últimas décadas. Por lo menos, podemos decir que este tipo de integración no consiguió la transformación esperada por los modelos en el comercio intra-sud-americano. El carácter abierto y orientado hacia afuera, dominó el padrón de inserción en la economía mundial. Esta característica no se modificó durante los años 2000-2009.

Conviene estudiar ahora las relaciones con tres países (o grupo de países en el caso de la Unión Europea): EEUU, Unión Europea y China. Los dos primeros son regiones más desarrolladas (menos heterogeneidad estructural y social y potencias tecnológicas y militares). En cuanto a China, viene modificando el comercio mundial y para algunos autores, el orden económico mundial desde los años 2000.

- Comercio de America del Sur con los EEUU y la Unión Europea: el padrón de comercio con los países del centro (EEUU y Unión Europea) es diferente ya que la mayoría de las exportaciones hacia la Unión Europea desde America del Sur son de productos primarios, mientras que para los EEUU, la proporción es de $30 \%$ para cada una de las categorías (primarios e industrializados ${ }^{(12)}$ ).

- Con los EEUU: las exportaciones de bienes de alta tecnología aumentaron de menos de $20 \%$ en 1985 a casi 40\% en 2001, para finalmente estabilizarse con alrededor de 30\% en los años 2003-2009. Los bienes intensivos en trabajo y recursos naturales que representaban cerca del 15\% de las exportaciones entre 1985-2003 aumentaron a 25\% en 2009. En lo que se refiere a las importaciones, $70 \%$ son de bienes industrializados (y $10 \%$ primarios) de los cuales $50 \%$ son de alta tecnología y $40 \%$ de tecnología media. Cuando analizamos los saldos, observamos tres períodos: i) 1985-1997: déficits importantes y crecientes en bienes industrializados de hasta 30 billones de dólares en 1997; ii) 1998-2003: reducción de este déficit hasta prácticamente 5 billones de dólares; iii) 20042009: fuerte y rápido aumento del déficit que llegó hasta 20 billones de dólares en 2008 y 2009. El saldo de bienes primarios de América del Sur con los EEUU es ligeramente superavitario de 1985-2006 y tiende a reducirse desde 2007.

- Con la Unión Europea: 70\% de las exportaciones son productos primarios. Las importaciones están compuestas de $90 \%$ de bienes industrializados de alta (casi 50\%) y media (40\%) tecnologías. El saldo es superavitario en bienes primarios (en aumento desde 2003 con 60 billones de dólares en 2008) y deficitario en bienes industrializados (más de 40 billones también en 2008). El padrón primo-exportador de América del Sur con la Unión Europea se mantuvo a lo largo del período estudiado y parece haberse consolidado, dejando las tentativas y discursos promovidos por la nueva CEPAL neo-estructuralista de los años 1990-2010 y en gran medida frustrados.

(12) Notemos que para llegar a 100\% faltan las categorías "no clasificados" que no aparecen en esta clasificación por ser productos para los cuales los mercados no aumentaron mucho durante las últimas dos o tres décadas (o sea son productos poco dinámicos no tomados en consideración en esta clasificación), o por ser productos de tipo "combustibles" que tampoco son considerados en esta metodología (ver UNCTAD, 2002, p. 87). 
- $\quad$ Comercio de America del Sul con China: el cambio se da al final de los años 1980 cuando los bienes primarios llegan a representar la mayor parte de las exportaciones de America del Sur (más de 80\%). El nivel tecnológico de los menos de $10 \%$ de bienes industrializados exportados a China es irrisorio. En cambio, en las importaciones de América del Sur provenientes de China, en el mismo periodo, más del 90\% de los bienes son productos industrializados y más de $40 \%$ de estos son de alta tecnología. Los bienes industrializados intensivos en trabajo y en recursos naturales importados desde China por América del Sur, se redujeron a partir de 1992 llegando a menos del 20\% del total de los bienes industrializados. El saldo comercial en bienes primarios viene creciendo a partir del 2002-2003 y llegó casi a 40 billones de dólares en 2009. Por otra parte, el saldo de los bienes industrializados ( $50 \%$ en alta tecnología) tuvo una evolución inversa alcanzando un déficit de más de 40 billones de dólares en 2008-2009. Es decir que con el nuevo principal socio comercial de America del Sur, el comercio mostró un padrón de comercio reprimarizado desde fines de los años $1980^{(13)}$.

\section{CONCLUSIÓN}

El presente estudio sobre el comercio de America del Sur durante el período 19852009 tiende a mostrar que el padrón de inserción en la economía mundial se mantuvo prácticamente inalterado. Si bien es verdad que el nivel tecnológico del comercio (exportaciones e importaciones) aumentó a lo largo del período, a nivel mundial, América de Sur se encuentra detrás de muchas regiones en desarrollo, como Asia ${ }^{(14)}$. No se puede negar que, a escala mundial, una mayor integración de las cadenas productivas de valor. Sin embargo, en el caso de América del Sur, gran parte del aumento del nivel tecnológico de sus exportaciones se debe más bien al aumento de las importaciones de bienes de elevado nivel tecnológico y a la presencia de ETN en la región que a una capacidad endógena de generar y producir bienes de alto nivel tecnológico. La capacidad de creación, mantenimiento y desarrollo de una base científico-tecnológica a nivel regional es todavía

(13) Así, puede existir un "Efecto commodities" como observa la UNCTAD : "In developing economies that are still highly dependent on the production and export of primary commodities, the link between growth and employment creation can be quite loose. This is because short-term growth is often influenced more strongly by movements in internationally determined prices for primary commodities than by an expansion in the volume of domestic output. Strong increases in commodity prices, as witnessed during the period 2002-2008, can lead to income growth without an increase in real output, and thus do not result in higher employment in the commodities sector. To the extent that higher commodity prices increase profits in that sector, they tend to have a very small impact, if any, on domestic demand, and thus on employment. However, to the extent that rising commodity prices translate into higher wages (or larger fiscal revenue and expenditure), they could have the same effect as productivity growth resulting from technological innovation: they could boost demand and employment in other parts of the economy. The latter situation is rare because of the frequent monopoly position of capital owners in the primary sector, especially in mining, and the particularly weak position of labour. Transforming productivity gains resulting from commodity price increases into a sustained process of growth and employment throughout the economy would require changing this situation to ensure that higher prices or productivity growth in the primary sector translate into greater domestic demand and/or more investment." (UNCTAD, 2010, p. 85-86.)

(14) La misma conclusión puede ser observada en lo que se refiere al sector de servicio. Mismo no siendo el tema central de este trabajo, recomendamos la lectura del los trabajo de la Red Mercosur sobre este tema: Red Mercosur (2010b). 
muy débil en América del Sur. Esta dificultad fue apuntada anteriormente por autores de diversos enfoques y, cuando comparamos los niveles de gastos en I \& D en función del PIB en Asia y en América del Sur, comprendemos que se trata de un gran desafío. En la década del 2000, Asia gastaba entre 1,5\% y 2,5\% de su PIB en I \& D, mientras que América del Sur solamente entre 0,5\% y 0,7\% del PIB de la región ${ }^{(15)}$.

El proyecto de una Agencia de Innovación a escala sud-americana es un desafío fundamental para la definición de una estrategia alternativa y progresista de integración económica regional. La reciente reunión del Consejo Andino de Ciencia y Tecnología en julio de 2010 fue una señal interesante que pretende definir una agenda de trabajo relativa a la formulación de una política comunitaria en Ciencia y Tecnología, coordinando acciones conjuntas entre países andinos, actualizando los programas de investigación y formación y creando un fondo para financiar proyectos (CEPAL, 2010, p. 126). Está claro que un tal proyecto tiene un costo elevado y necesita una visión estratégica. La UNASUR podría colocar este tema como elemento estratégico prioritario con recursos y financiamientos asociados. La iniciativa de promover un Centro de Investigación Regional de la CAN en 2010 es fundamental para pensar de manera estratégica y dinámica la inserción de America del Sur en la economía mundial.

Las características del régimen de acumulación financiarizado y excluyente, predominante en América del Sur, parecen no permitir que se desarrolle en la División Internacional del Trabajo, un modelo de acumulación más homogéneo y con menor desigualdad, ni tampoco una inserción menos predadora socialmente y ambientalmente.

\section{BIBLIOGRAFÍA}

ALADI (2008). Seguimiento de las actividades en el marco de la Iniciativa para la Integración de la Infraestructura Regional Sudamericana (IIRSA), el Plan Puebla-Panamá (PPP), la Unión de Naciones Sudamericanas (UNASUR) y la Iniciativa de Transporte del Hemisferio Occidental (ITHO). Informe del primer semestre (ALADI/SEC/di 2160), 15 de junio.

ALADI, Boletín Electrónico Comercio Exterior en Cifras, varios números. Disponível em: <http:// www.aladi.org/nsfaladi/arquitec.nsf/vsitioweb/boletinaladi>.

BASUALDO, Eduardo; ARCEO, Enrique (comp.) Neoliberalismo y sectores dominantes. Tendencias globales y experiencias nacionales. Buenos Aires: Clacso, 2006.

BID. Economic and social progress in Latin America: 2002 report; Beyond border, the new regionalism in Latin America. Washington D.C.: BID, 2002.

COMUNIDAD ANDINA DE NACIONES (CAN). Disponível em: <http://www.comunidadandina. org/>.

CARCANHOLO, Marcelo Dias. Neoconservadorismo com Roupagem Alternativa: a Nova Cepal dentro do Consenso de Washington. In: Castelo, R. (Org.) Encruzilhadas da América Latina no Século XXI. Rio de Janeiro: Pão e Rosas, 2010.

CEPAL. Panorama de la inserción internacional de América Latina y el Caribe 2009-2010: Crisis originada en el Centro y recuperación impulsada por las economías emergentes. 216 pp. Santiago de Chile: CEPAL, 2011.

(15) Ver Katz, 2001, p. 116-120, Palma, 2010, Perez, 2001 o desde un punto de vista más crítico Varsavsky, 1974. 
Panorama de la inserción internacional DE AMÉRICA LATINA Y EL CARIBE 2008-2009. Santiago de Chile: CEPAL, 2011.

CEPAL. 2009. Panorama da Inserção de America Latina CEPAL. Disponível em: <http://www. eclac.org/publicaciones/xml/6/36906/PANORAMA_DE_LA_INSERCION_INTERNACIONAL_2008_2009_vf.pdf>.

CEPAL. 2008. Inseción economía mundial 2007 y tendencias 2008. Disponível em: <http://www.eclac. org/publicaciones/xml/9/34329/PANORAMA_INSERCION_INTERNACIONAL_2008_vf.pdf>.

CEPAL. El regionalismo abierto en America Latina y el Caribe; la integración económica al servicio de la Transformación Productiva con Equidad. Santiago de Chile: CEPAL, 1994.

CEPAL, BADECEL. Disponível em: <http://www.eclac.org/Comercio/paninsal/Anexo2008_2009/ espanol/portada.htm>.

GAMBINA Julio. 2011. Un modelo en discusión, en p. 12, "Debate: Que integración regional conviene?" Disponible en:<http://www.paginal2.com.ar/diario/economia/2-170443-2011-06-20.html>. IIRSA, Planificación Territorial Indicativa: Cartera de Proyectos IIRSA 2009. Disponível em:<http:// www.iirsa.org/BancoConocimiento/L/lb09_cartera_de_proyectos_iirsa_2009/lb09_cartera_de_ proyectos_iirsa_2009.asp?CodIdioma=ESP $>$.

INTAL-BID, Informe MERCOSUL 14. Disponível em: <http://www.iadb.org/intal/aplicaciones/ uploads/publicaciones/p_Informe_MERCOSUL_14.pdf>.

KATZ, Jorge. 2000. Structural reforms, productivity and technological change in Latin America. Libros de la CEPAL n. 64, Santiago du Chili.

MARINI, Rui Mauro. Dialéctica de la Dependencia. Serie Popular Era, 3. edição, 1977. México.

PALMA, José Gabriel. 2010. Why has productivity growth stagnated in most Latin-Amercian countries since the neo-liberal reforms, CWPE 1030. Disponível em: <http://www.econ.cam.ac.uk/dae/ repec/cam/pdf/cwpe1030.pdf>.

PEREZ, Carlota. Technological change and opportunities for development as amoving target Revista da CEPAL, n. 75, 2001.

RED MERCOSUR. Los Impactos de la Crisis Internacional en America Latina y las Alternativas de políticas. Serie policy brief, n. 2/10 - diciembre 2010a.

ROMER, Paul. El Cambio tecnológico endógeno. In: Journal of Political Economy, Chicago, v. 98, n. 5, 1990.

SALAMA, Pierre. Brésil, Bilan économique, succès et limites. In: Problèmes d'Amérique Latine. Paris, n. 78, 2010.

Du productif au financier et du financier au productif en Asie et en Amérique latine. In: Conseil d'Analyse Economique, Développement, Paris, 2000.

2010. Estratégias de Desenvolvimento e Inserção da América Latina na Economia Mundial. Os estruturalistas e neoestruturalistas da Cepal: uma abordagem crítica. In: CASTELO BRANCO, Rodrigo. (Org.) Encruzilhadas da América Latina no Século XXI. Rio de Janeiro: Pão e Rosas, 2010.

SALUDJIAN, Alexis. Del Mercosur al ALCA: Críticas al modelo liberal de Nuevo Regionalismo de la BID. In: Problemas del Desarrollo. México, v. 36, n. 141, p. 9-25, Abr.-Jun., 2005.

SANTOS (dos) Theotonio. A Teoria da Dependência: balanços e perspectivas. Rio de Janeiro: Civilização Brasileira, 2000.

SELA. Informe sobre el Proceso de Integración Regional. 2009-2010, Caracas, Octubre, 2010. 
UNASUL. Disponível em: <http://www.pptunasur.com/>.

UN COMTRADE, base de dados, acesso Novembro 2010.

UNCTAD, Trade and Development report, 2002. Disponível em: <http://www.unctad.org/en/docs/ tdr2002_en.pdf>.

UNCTAD, 2010, Trade and Development report, Employment, globalization and development. Disponível em: <http://www.unctad.org/Templates/Download.asp?docid=13740\&lang=1\&intIte $\mathrm{mID}=1397>$.

VARSAVSKY, Oscar. Estilos Tecnológicos. Sd: ed. Periferia, 1974.

\section{Anexo III - Saldos do Comércio}

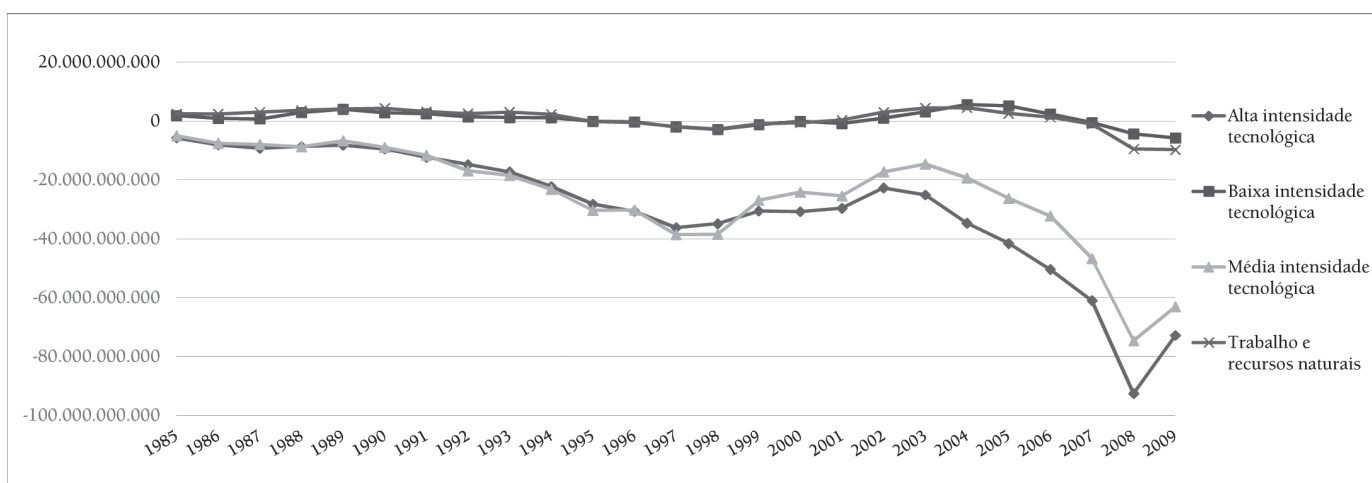

\section{ANEXO 1.1 SALDO DE EXPORTAÇÃO DE BENS INDUSTRIALIZADOS DA AMÉRICA DO SUL PARA O MUNDO, POR NÍVEL TECNOLÓGICO}

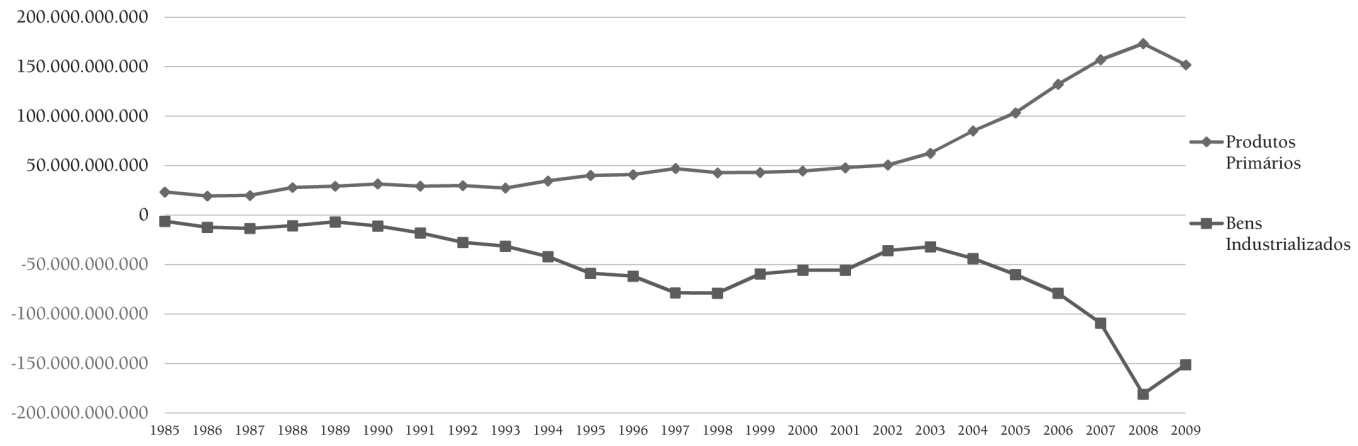

ANEXO 1.2 SALDO DE EXPORTAÇÕES DA AMÉRICA DO SUL PARA O MUNDO, POR TIPO DE PRODUTO 


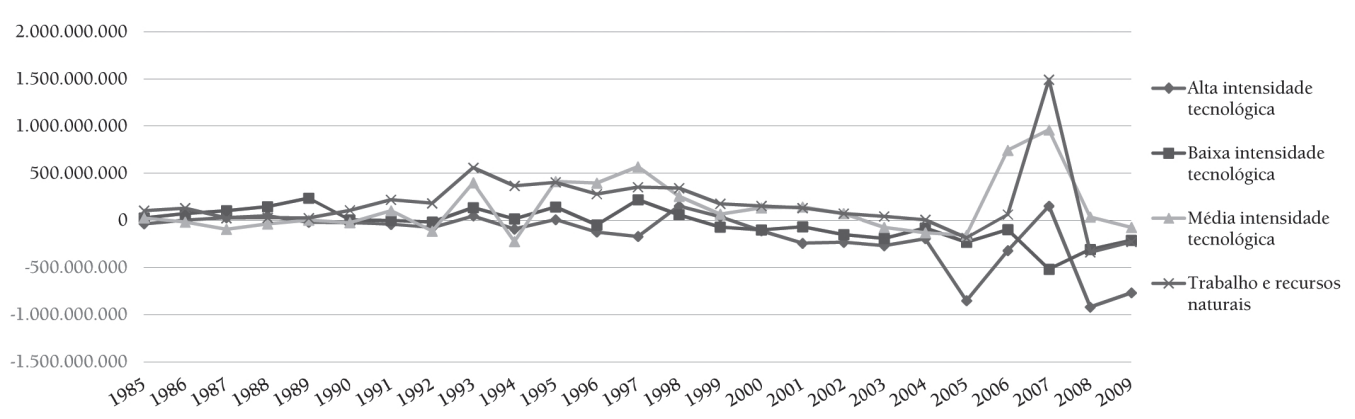

ANEXO 2.1 SALDO DE EXPORTAÇÕES DE BENS INDUSTRIALIZADOS DA AMÉRICA DO SUL PARA A AMÉRICA DO SUL, POR NÍVEL TECNOLÓGICO

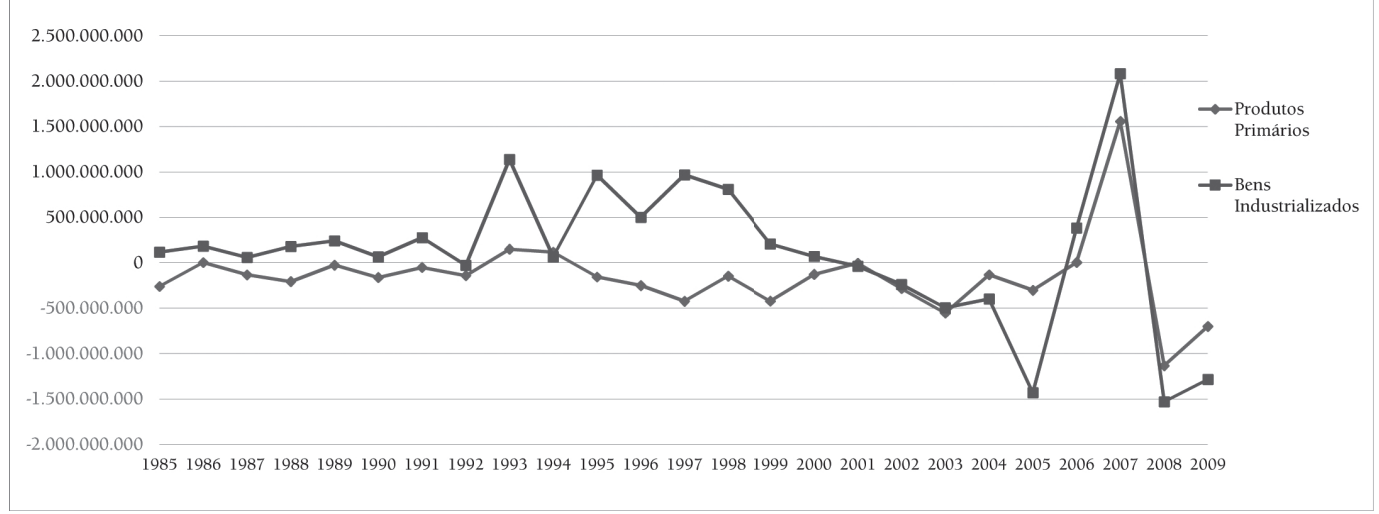

ANEXO 2.2 SALDO DO COMÉRCIO DA AMÉRICA DO SUL PARA A AMÉRICA DO SUL, POR TIPO DE PRODUTO 


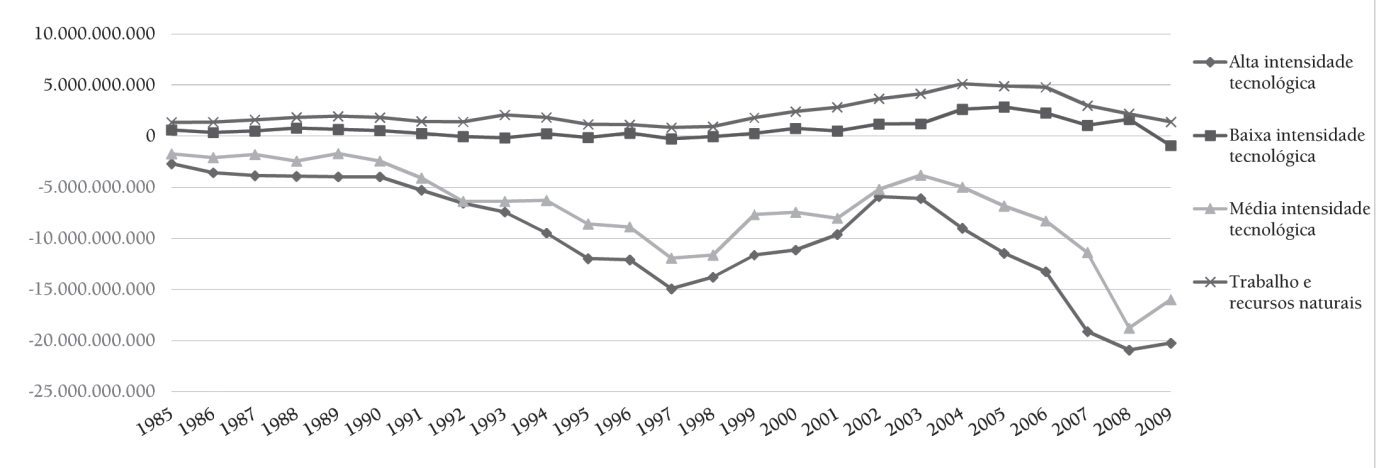

ANEXO 3.1 SALDO DO COMÉRCIO DE BENS INDUSTRIALIZADOS DA AMÉRICA DO SUL PARA OS EUA, POR NÍVEL TECNOLÓGICO

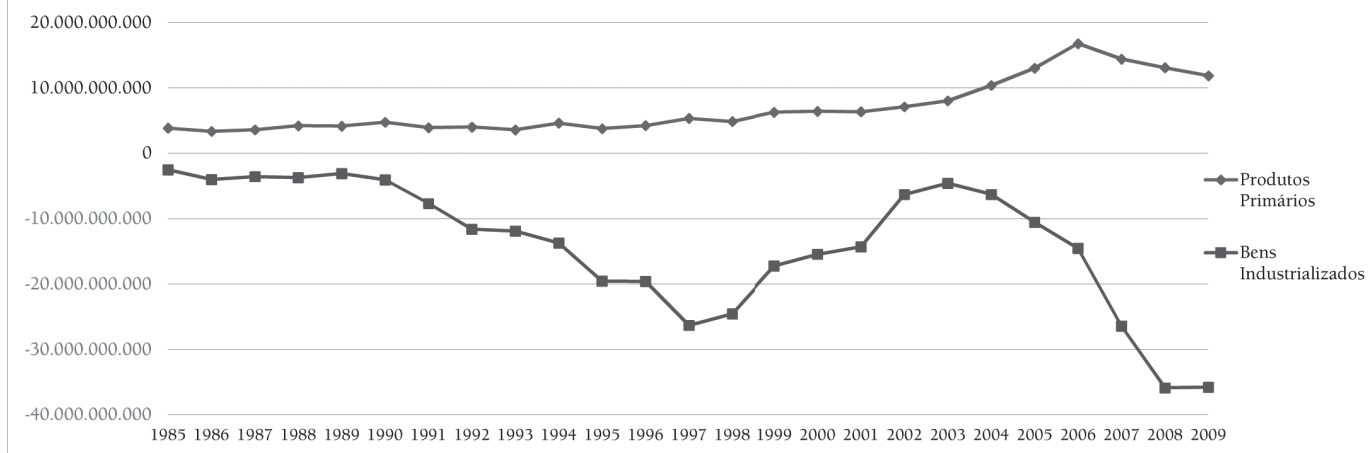

ANEXO 3.2 SALDO DO COMÉRCIO DA AMÉRICA DO SUL PARA OS EUA, POR TIPO DE PRODUTO 


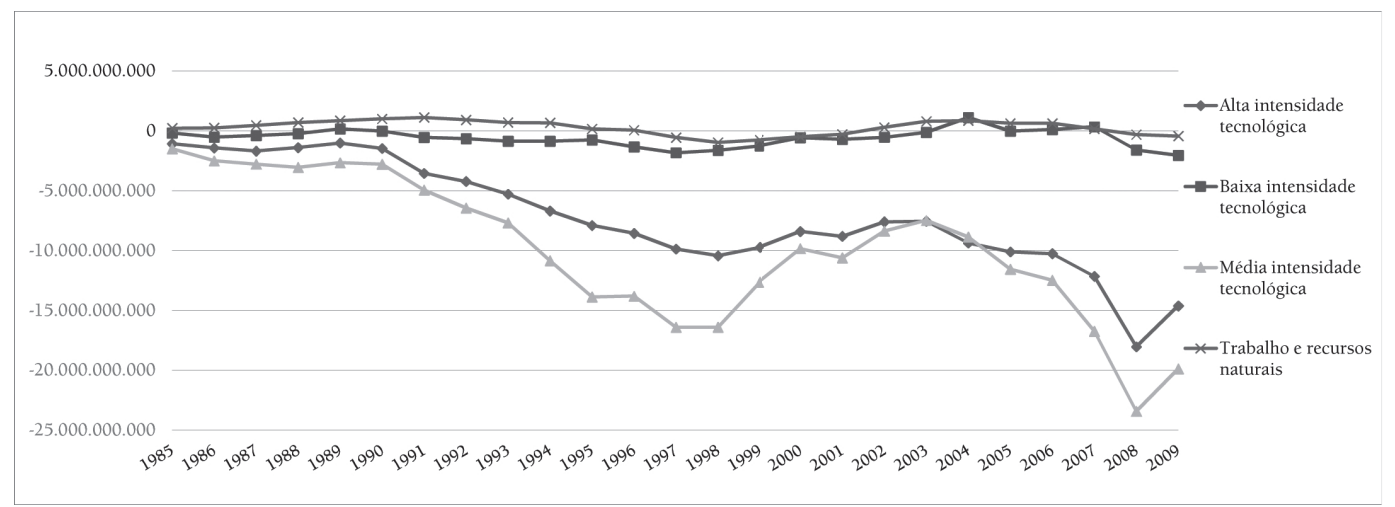

ANEXO 4.1 SALDO DE EXPORTAÇÕES DE BENS INDUSTRIALIZADOS DA AMÉRICA DO SUL PARA A UNIÃO EUROPEIA, POR NÍVEL TECNOLÓGICO

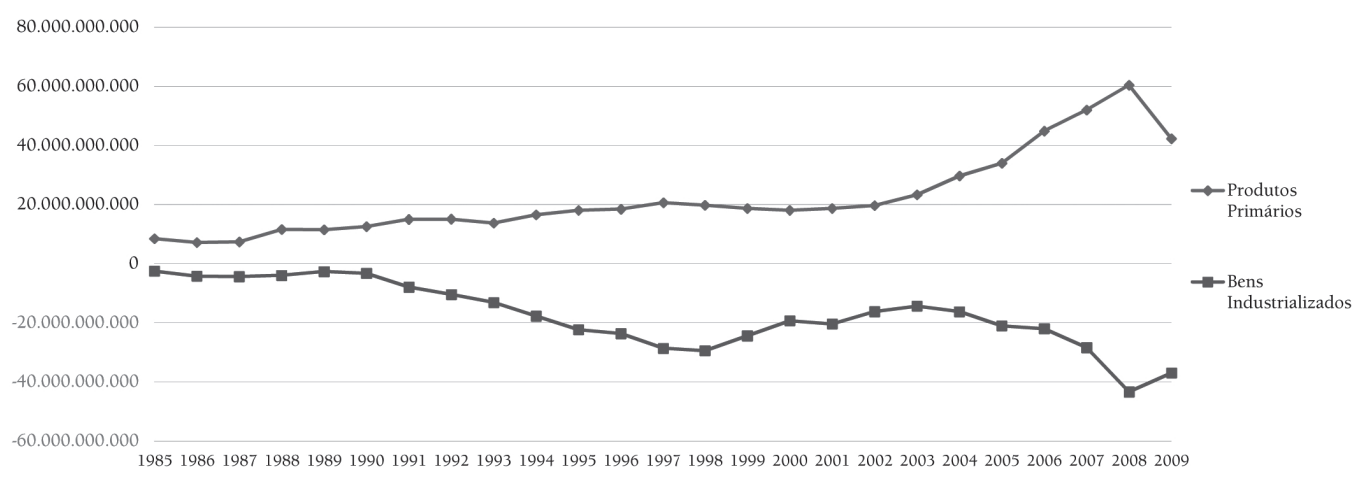

ANEXO 4.2 SALDO DO COMÉRCIO DA AMÉRICA DO SUL PARA A UNIÃO EUROPEIA, POR TIPO DE PRODUTO 


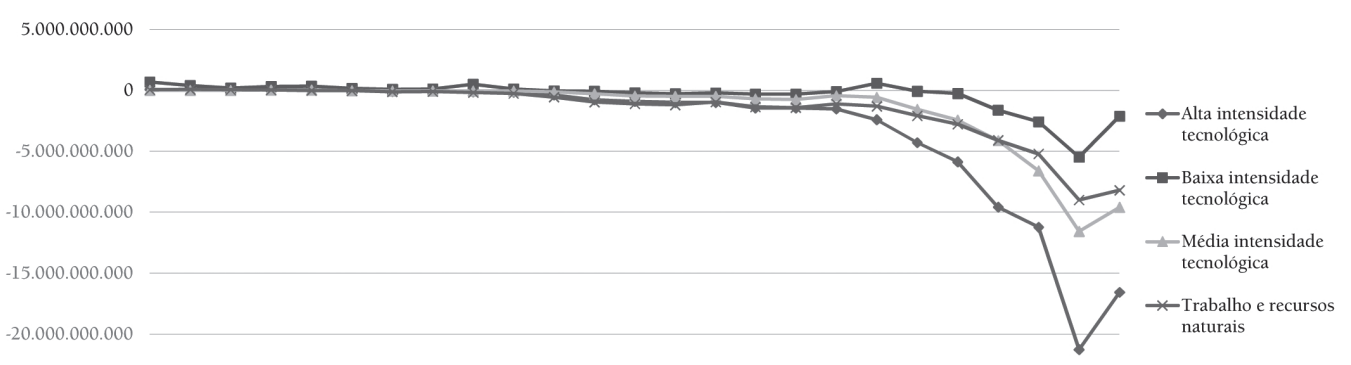

$-25.000 .000 .000$

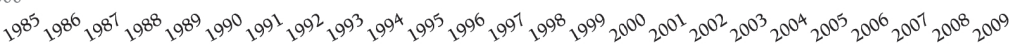

ANEXO 5.1 SALDO DE EXPORTAÇÕES DE BENS INDUSTRIALIZADOS DA AMÉRICA DO SUL PARA A CHINA, POR NÍVEL TECNOLÓGICO

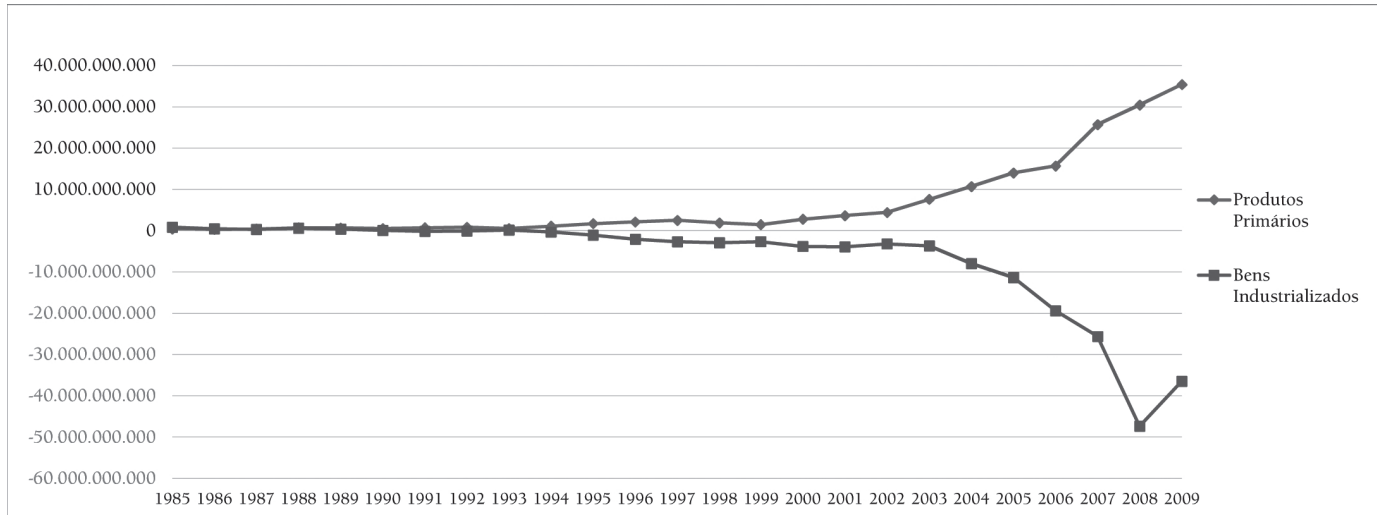

ANEXO 5.2 SALDO DO COMÉRCIO DA AMÉRICA DO SUl PARA A CHINA, POR TIPO DE COMMODITY 